Systematic Review

\title{
Anterior Cingulotomy for the Treatment of Chronic Intractable Pain: A Systematic Review
}

Justin Sharim, MD', and Nader Pouratian, MD, PhD 2,3

From: ${ }^{1}$ David Geffen School of Medicine at UCLA, Los Angeles, CA; ${ }^{2}$ Department of Neurosurgery, David Geffen School of Medicine at UCLA, Los Angeles, CA; ${ }^{3}$ Neuroscience Interdepartmental Program, and Brain Research Institute, David Geffen School of Medicine at UCLA, Los Angeles, CA

Address Correspondence: Nader Pouratian, MD, PhD David Geffen Schoool of Medicine at UCLA 300 Stein Plaza, Ste 562 Los Angeles, CA 90095 E-mail:

npouratian@mednet.ucla.edu

Disclaimer: There was no external funding in the preparation of this manuscript. Conflict of interest: Each author certifies that he or she, or a member of his or her immediate

family, has no commercial association (i.e., consultancies, stock ownership, equity interest, patent/licensing arrangements, etc.) that might pose a conflict of interest in connection with the submitted manuscript.

Manuscript received: 03-25-2016 Revised manuscript received: 03-31-2016 Accepted for publication: 05-18-2016

Free full manuscript: www.painphysicianjournal.com
Background: Anterior cingulotomy for chronic pain aims to modulate patients' attention or emotional reaction to pain rather than to modulate pain intensity.

Objectives: To evaluate the clinical efficacy, both short- and long-term, of anterior cingulotomy in the treatment of chronic pain.

Study Design: Systematic review.

Setting: This systematic review assessed studies reporting anterior cingulotomy for the treatment of chronic pain.

Methods: A systematic search of Web of Science, Scopus, PubMed, and PsychINFO was performed using both key words and controlled vocabulary. Articles included in this review included peerreviewed articles describing clinical outcomes or efficacy of cingulotomy in the treatment of chronic pain with minimum follow-up of 3 months for non-malignant and 2 weeks for malignant pain. Articles reporting cingulectomies or cingulotomy only as combined with other ablative procedures were excluded, as were individual case reports.

Results: A total of 11 articles encompassing 224 patients are included in the review, with age ranging 22 to 85 (mean: 56) years at the time of the operation, 59\% of which were men. Greater than $60 \%$ of patients across all studies were reported to have significant pain relief post-operatively as well as at one year after surgery. Common transient adverse effects included urinary incontinence and confusion/disorientation, subsiding within days postoperatively. Serious/permanent adverse effects included seizure in less than $5 \%$, hemiparesis in less than $1 \%$, and personality change in less than $1 \%$ of operations reported across all studies, all of which occurred primarily in operations where magnetic resonance (MR)-guidance was not used.

Limitations: The limitations of this systematic review include the lack of studies other than observational reports and the inevitable heterogeneity between included studies.

Conclusions: Despite decreased utilization in recent years, anterior cingulotomy is an effective neurosurgical intervention in the treatment of pain and carries little risk of permanent or serious adverse effects.

Key words: Anterior cingulotomy, chronic pain, stereotaxis, systematic review, pain, cingulate gyrus, cingulotomy, intractable pain

Pain Physician 2016; 19:537-550
D ain is the most common complaint of patients seeking medical care (1), and can be debilitating to the degree of preventing normal everyday function. However, relief of chronic pain in cancer patients or in patients with non-neoplastic sources of pain can be difficult to achieve and maintain (2). Nonsteroidal anti-inflammatory drugs, opiates, and nonnarcotic analgesics remain the mainstay of treatment; 
however, the dose of such medications can be increased only to the extent to which the benefit of pain relief outweighs the drug-induced adverse effects. When pain thus proves to be refractory to such medications, neuromodulatory and neurosurgical interventions may play a role (3-6).

Pain has been described in 3 dimensions: sensory (pain intensity), affective (pain unpleasantness), and cognitive $(7,8)$. The anterior cingulate cortex (ACC) has been shown to be involved in the perception of pain, particularly the affective component $(9,10)$. The ACC is located in the medial part of the cerebral hemispheres partially circumscribing the corpus callosum. Unlike the posterior cingulate cortex, the ACC is part of the limbic system, receiving inputs from other limbic structures such as the amygdala, and projecting outputs to the periaqueductal grey and brainstem. Its role in emotion has led to it being targeted in ablative procedures treating medically refractory psychiatric illnesses including obsessive-compulsive disorder, depression, and severe anxiety (11-16).

For decades, anterior cingulotomy has been performed in the treatment of chronic medically refractory pain $(1,2,9,15,17-23)$. Anterior cingulotomy is thought to function not by modulating the sensation of pain intensity, but rather, the patient's attention or emotional reaction to the pain $(1,9,17)$. The affective component of afferent pain fibers has been shown to be relayed from midline thalamic nuclei to the cingulate gyrus $(9,24,25)$. Experimental and functional imaging studies have shown a direct role of the cingulate gyrus in chronic pain processing (26-29). Moreover, in vitro studies have demonstrated long-term potentiation of excitatory synapses within the ACC in response to injuries (30). Such potentiation has been shown to continue in the absence of input from the periphery, implicating the ACC to play a role in chronic pain. Together these results provide an anatomic, functional, and cellular rationale for ablative lesions of the ACC in treating medically refractory chronic pain.

In recent years, ablative lesions such as anterior cingulotomy have fallen out of favor in deference to other neuromodulatory therapies such as spinal cord and deep brain stimulation as well as intrathecal therapies $(4,6)$. However, there still remain patients who fail to respond to these therapies $(4,31)$. Therefore, understanding the role and efficacy of ablative procedures is critical in defining the comprehensive approach to pain management. The goal of this systematic review is to evaluate the clinical efficacy, both short- and long-term, of anterior cingulotomy in the treatment of chronic medically refractory pain.

\section{Methods}

The methodology used in this review was in accordance with Preferred Reporting Items for Systematic Reviews and Meta Analyses (PRISMA) guidelines, Agency for Healthcare Research and Quality (AHRQ) recommendations (www.effectivehealthcare.ahrq.gov), and formal review processes derived from evidence-based systematic reviews $(32,33)$.

\section{Data Source and Search}

A systematic search of the following electronic databases was performed through October 2015: Web of Science, Scopus, PubMed, and PsychINFO. Key words and controlled vocabulary (i.e., MeSH terms) were employed using Boolean "and" and "or" arguments. Key words used included "pain," "chronic pain," "cingulotomy," "cingulumotomy," and "cingulectomy"; controlled vocabulary search terms included "pain" and "psychosurgery." A representative search is as follows: (((pain[MeSH Terms]) OR pain OR "chronic pain") AND ("psychosurgery" [MeSH Terms] OR cingulotomy OR cingulumotomy)). No language restrictions were employed. Conference proceedings were included. Additional articles were hand searched through review of references cited within articles. Review articles were used only to extract any additional articles not found within the original search and were not included as separate articles themselves within the analysis. Articles prior to 1975 found through such citation mapping were hand searched within the Biomedical Research Library at the University of California at Los Angeles.

\section{Study and Patient Selection}

We included peer-reviewed articles that described clinical outcomes or efficacy of cingulotomy in the treatment of chronic pain. To be included, reports must have included a minimum follow-up of at least 3 months for patients with nonmalignant sources of pain and 2 weeks for patients with malignant sources of pain (due to the shorter survival in this patient cohort). Articles reporting only on anterior cingulotomy in combination with other ablative procedures were excluded from the review, as were articles reporting cingulectomies. Some articles reported anterior cingulotomy combined with another ablative procedure in some patients but alone in others (20), and thus were included. From these articles, individual patients undergoing combined 
surgeries were excluded from the review. Individual case reports involving one or 2 patients were excluded from the analysis. Patients requiring reoperations were noted. For publications from the same authors, care was taken to not count individual patients twice. A summary of study inclusion and exclusion criteria is provided in Table 1.

\section{Data Synthesis and Analysis}

Data were summarized based on study design (sample size, reporting quality), patient demographics (age, gender, cause of pain), intervention characteristics (lesion type, guidance, location), and method of quantifying pain relief. Given that included articles quantified pain relief using multiple different methods and often stratified the degree of pain relief categorically (e.g., "good," "excellent"), we defined "significant" pain relief as that which comprised relief of at least a $50 \%$, or for articles stratifying patients categorically, as that falling under any categories with median pain relief of at least $50 \%$, or otherwise if not reported, left to the discretion of the original authors.

Of the articles with patients requiring reoperation, some did not discriminate between reoperation and original surgery in the presentation of overall results; thus, overall outcomes presented here included reoperations - thus intent to treat analysis. In the calculation of effect of lesion location on pain relief, some articles provided ranges of lesion location midpoints, while others provided a single midpoint value. For the articles providing ranges, a mean of the endpoints of the range was used.

\section{Results}

\section{Patient Demographics}

A total of 11 articles encompassing 224 patients are included in the review (Fig. 1). Of the articles that provided age ranges for patients $(1,2,15,18,19,21-23)$, age ranged from 22 to 85 years at the time of operation. Of these articles, 7 provided mean or median ages of patients included in the study $(1,2,15,18,19,22,23)$. The weighted mean (based on number of patients included in the analysis) of the means or medians provided was 56 years of age at the time of operation. Seven articles provided information about the gender of patients $(1,15,18,19,21-23)$, to include a total of 147 patients. Of these, $87(59 \%)$ were men and $60(41 \%)$ were women.

Due to possible differences between neoplastic and non-neoplastic sources of pain, we performed additional
Table 1. Study selection criteria.

\begin{tabular}{|l||}
\hline Inclusion \\
\hline Dx of chronic pain intractable to medication \\
\hline Cingulotomy \\
\hline Pain quantification before and after cingulotomy \\
\hline Adult patient (age $\geq 18$ years) \\
\hline Exclusion \\
\hline All cingulotomies exclusively combined w/ other intervention \\
\hline Case report \\
\hline Open cingulectomy \\
\hline FU $<2$ weeks for neoplastic or $<3$ months for non-neoplastic pain \\
\hline
\end{tabular}

$\mathrm{Dx}=$ diagnosis; $\mathrm{FU}=$ follow-up

analyses of each of these subsets. All articles provided information regarding source of pain (Table 2). Nine articles included patients with pain of neoplastic origin, 2 articles of which included only patients with pain of neoplastic origin, encompassing 97 patients. Likewise, 9 articles included patients with non-neoplastic sources of pain, 2 articles of which exclusively included nonneoplastic sources, encompassing 127 patients.

In all articles, patient selection criteria included severe disabling pain that was refractory to medication. Many of the articles $(9,15,17,18)$ described emotional factors (e.g., diagnosis of depression) existing in conjunction with pain, which were believed to be contributing to the pain symptoms or were themselves attributed to, at least in part, the unrelenting pain. Unrelenting chronic pain itself is empirically known to contribute to depression (34). Moreover, since many patients were in the terminal stages of cancer, comorbid depression or anxiety was deemed understandable by the original authors (35). In such cases however, it was made clear that cingulotomy was done to relieve suffering from the pain, and not for the treatment of psychiatric illness.

\section{Time-dependent Outcomes}

Of the total 224 patients included in this analysis, $149(67 \%)$ had significant relief post-operatively. Of 98 patients with cancer pain, $66(67 \%)$ had significant pain relief post-operatively. Of 127 patients with pain of non-neoplastic origin, $83(65 \%)$ had significant pain relief post-operatively (Table 3A, 3B, 4).

A total of 156 (out of 224) patients had followup reported for at least 3 months, of which 87 (56\%) had significant pain relief. Since short-term mortality was significantly higher in cancer patients, follow-up for these patients were generally shorter than that of 


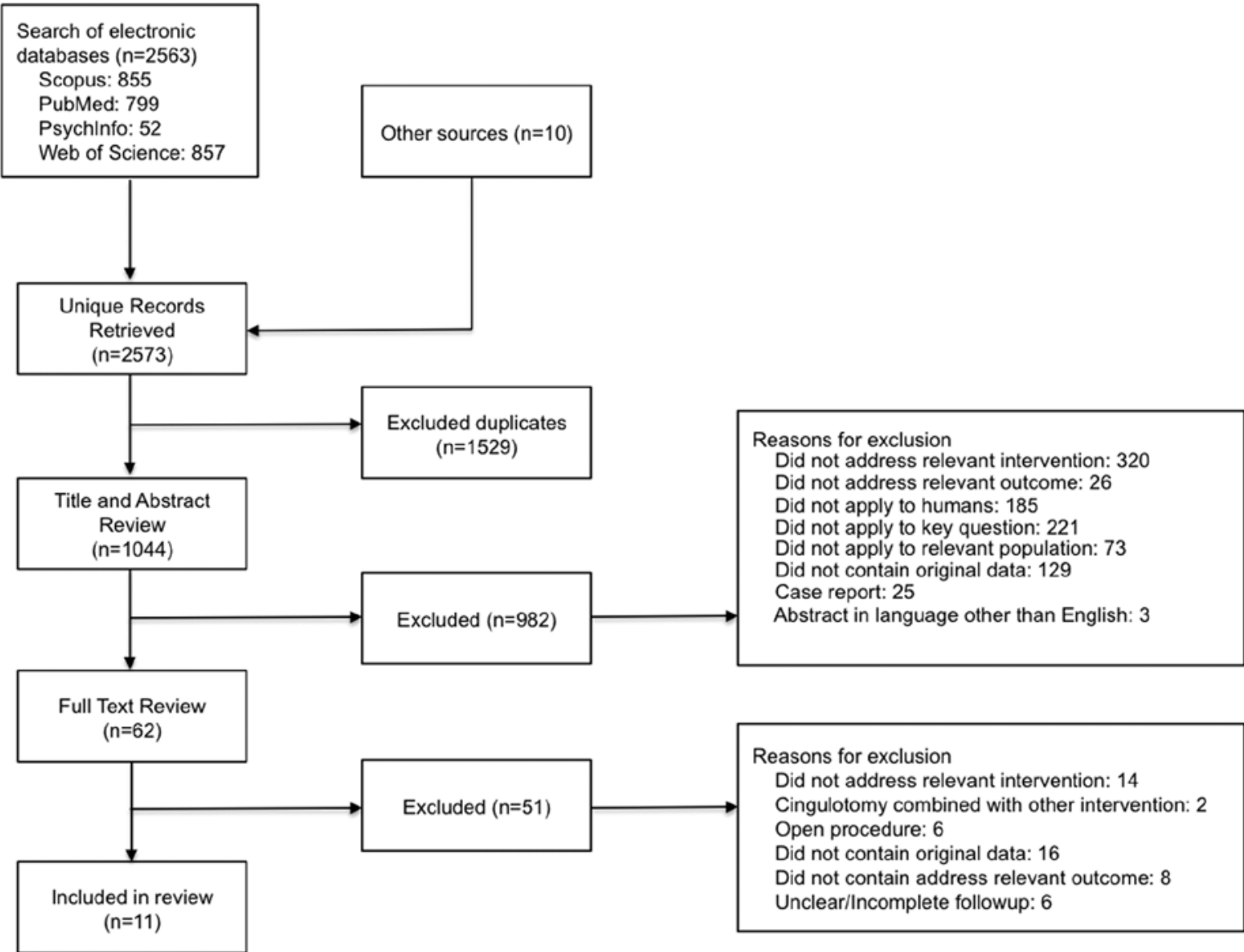

Fig 1. PRISMA flow chart diagram for included studies.

patients with non-neoplastic sources of pain. Forty-four patients with cancer pain had confirmed follow-up to at least 3 months, of whom $23(52 \%)$ had significant pain relief. One hundred and twelve patients with nonneoplastic sources of pain had follow-up time to at least 3 months duration, of whom 64 (57\%) had significant pain relief.

A total of 94 patients had follow-up to at least 6 months post surgery, of which $59(63 \%)$ were reported to have significant pain relief. Of 20 cancer patients who had follow-up to at least 6 months, $12(60 \%)$ had significant pain relief. Likewise, 47 of $74(64 \%)$ patients with non-neoplastic pain with at least 6 months followup reported significant pain relief.

Amongst 82 patients with at least one year of follow-up, $53(65 \%)$ reported pain relief, including 6 of 9 cancer patients (67\%) and 47 of $73(64 \%)$ patients with non-neoplastic pain. A summary of this data is provided in Table 4.

The outcomes described above are based on response rates amongst all patients with available followup at each time point, implicitly assuming that response rates are equal amongst patients with and without follow-up. The majority of patients with neoplastic pain had short follow-up due to death attributable to the primary malignancy. However, for patients with non-neoplastic pain, patients were generally lost to follow-up for reasons other than death. We therefore also evaluated the efficacy of anterior cingulotomy in patients with non-neoplastic pain assuming that patients with shortened follow-up that was not explicitly attributed to death were failures of treatment. Using 
Table 2. Patient population, operative technique, and quantification of pain.

\begin{tabular}{|c|c|c|c|c|c|}
\hline & $\begin{array}{c}\text { Patient } \\
\text { demographics }\end{array}$ & $\begin{array}{c}\text { No. of } \\
\text { Patients } \\
\text { (neoplastic } \\
\text { + non- } \\
\text { neoplastic) }\end{array}$ & Cause of Pain & Operative technique & $\begin{array}{c}\text { Pain } \\
\text { Quantification }\end{array}$ \\
\hline $\begin{array}{l}\text { 1. Foltz and } \\
\text { White, } 1962\end{array}$ & Not stated & $16(6+10)$ & $\begin{array}{l}\text { Neoplastic: head and neck pain } 2 \text {, } \\
\text { back and hip pain } 2 \text {, shoulder and } \\
\text { arm pain } 1 . \\
\text { Non-neoplastic: face pain } 2 \text {, vaginal- } \\
\text { perineal pain } 2 \text {, chest pain } 1 \text {, arm } \\
\text { causalgia } 1 \text {, "emotional" angina } 1 \text {, } \\
\text { atypical causalgia thigh stump } 1 \text {, } \\
\text { leg pain } 2\end{array}$ & $\begin{array}{l}\text { Unilateral (5 patients) } \\
\text { or bilateral (11 patients) } \\
\text { anterior cingulotomy } \\
\text { under fractional } \\
\text { pneumoencephalography } \\
\text { or air ventrigulography } \\
\text { guidance, with tips of } \\
\text { electrodes } 5-6 \mathrm{~mm} \text { above } \\
\text { superior ependymal of } \\
\text { frontal horns and } 1-2.5 \\
\mathrm{~cm} \text { posterior to tip of } \\
\text { frontal horns, } 1.3 \mathrm{~cm} \\
\text { from midline. Lesions } \\
\text { made with electrocautery } \\
\text { (coagulation), 20-30 } \\
\text { seconds }\end{array}$ & Clinical assessment \\
\hline $\begin{array}{l}\text { 2. Foltz and } \\
\text { White, } 1968\end{array}$ & Not stated & $35(11+24)$ & $\begin{array}{l}\text { Neoplastic: throat cancer } 1 \text {, tongue } \\
\text { cancer } 1 \text {, face and neck cancer } 1 \text {, } \\
\text { back-hip metastases } 2 \text {, shoulder/ } \\
\text { arm cancer } 1 \text {, phantom limb pain } 1 \\
\text { Non-neoplastic: headache } 1 \text {, } \\
\text { coccydynia 1, face pain } 3 \text {, vaginal- } \\
\text { perineal pain 2, chest pain 1, } \\
\text { "burning feet" } 1 \text {, causalgia } 3 \text {, } \\
\text { emotional angina 1, pancreatitis } \\
\text { 1, painful legs (parapresis and } \\
\text { paraplegia 3, thalamic face pain } 1 \text {, } \\
\text { hemi-body pain 2, chest hyperalesia } \\
\text { 1, lumbar arachnoiditis } 3\end{array}$ & $\begin{array}{l}\text { Unilateral ( } 6 \text { patients) } \\
\text { or bilateral ( } 29 \\
\text { patients) anterior } \\
\text { cingulotomy under } \\
\text { xray ventriculographic } \\
\text { guidance } 2.5 \mathrm{~cm} \\
\text { posterior to tip of frontal } \\
\text { horns. Lesions made by } \\
\text { radiofrequency generator } \\
45-50 \mathrm{C}\end{array}$ & $\begin{array}{l}\text { Clinical assessment, } \\
\text { with } 5 \text { tier system } \\
\text { (excellent = no } \\
\text { spontaneous } \\
\text { episodes of pain, } \\
\text { good = occasional } \\
\text { episode of pain, fair } \\
\text { = pain present but } \\
\text { not persistent, poor } \\
\text { = no change in pain, } \\
\text { worse = increased } \\
\text { pain complaints) }\end{array}$ \\
\hline $\begin{array}{l}\text { 3. Faillace et al., } \\
1971\end{array}$ & $\begin{array}{l}\text { Age: range } 42- \\
66 \text { (median } 51 \text { ) } \\
\text { Gender: } 4 \mathrm{M} 5 \mathrm{~F}\end{array}$ & $9(7+2)$ & $\begin{array}{l}\text { Neoplastic: Terminal cancer } \\
\text { (unspecified). } \\
\text { Non-neoplastic: Intractable back } \\
\text { pain. }\end{array}$ & $\begin{array}{l}\text { Stereotactic bilateral } \\
\text { anterior cingulotomy } \\
\text { done by radiofrequency } \\
\text { heat ablation, } 3.5-4 \mathrm{~cm} \\
\text { posterior to tip of frontal } \\
\text { horns. }\end{array}$ & $\begin{array}{l}\text { Clinical assessment } \\
\text { based on patient's } \\
\text { subjective report }\end{array}$ \\
\hline $\begin{array}{l}\text { 4. Hurt and } \\
\text { Ballantine, } \\
1974\end{array}$ & $\begin{array}{l}\text { Age: range } 22 \\
-85 \text {, mean } 56 \\
\text { years } \\
\text { Gender: } 43 \mathrm{M} \\
25 \mathrm{~F}\end{array}$ & $68(32+36)$ & $\begin{array}{l}\text { - Neoplastic: Carcinoma of mouth } \\
\text { (4), pharynx (2), larynx (3), lung } \\
\text { (7), colon (3), pancreas (3), uterus } \\
\text { (2),bladder (2). Melonoma (2). } \\
\text { Liposarcoma 1, osteosarcoma 1, } \\
\text { undifferentiated neoplasm 1 } \\
\text { - Non-neoplastic: Atypical facial } \\
\text { pain 1, central pain 1, phantom limb } \\
\text { pain 2, tabetic pain, postherpetic } \\
\text { facial pain 2, postherpetic } \\
\text { truncal pain 2, paraplegic pain } \\
\text { 2, arachnoiditis pain 8, pain of } \\
\text { uknown etiology (visceral) 6, pain } \\
\text { of unknown etiology (somatic) } 10\end{array}$ & $\begin{array}{l}\text { Stereotactic bilateral } \\
\text { anterior cingulotomy } \\
\text { under ventriculographic } \\
\text { guidance, } 2-4 \mathrm{~cm} \\
\text { posterior to tip of frontal } \\
\text { horns. Lesions made by } \\
\text { radiofrequency } 8 \text { watts for } \\
60-75 \text { seconds. }\end{array}$ & $\begin{array}{l}\text { Review of medical } \\
\text { records, assigning } \\
5 \text { point scale. In } 36 \\
\text { patients alive ( } 34 \\
\text { non-noeplastic } \\
\text { and } 2 \text { neoplastic), } \\
\text { questionnaire sent } \\
\text { (complete (100\% } \\
\text { relief), marked (70- } \\
90 \% \text { relief), moderate } \\
\text { ( } 40-60 \% \text { relief), } \\
\text { slight (10-30\% relief), } \\
\text { none (0\%relief) as } \\
\text { substitute. } 28 \text { replied }\end{array}$ \\
\hline $\begin{array}{l}\text { 5. Voris and } \\
\text { Whisler, } 1975\end{array}$ & $\begin{array}{l}\text { Not stated } \\
\text { explicitly }\end{array}$ & $16(5+11)$ & $\begin{array}{l}\text { Neoplastic: Head and neck pain } 24, \\
\text { trunk pain } 6 \text {, extremities pain } 5, \\
\text { generalized pain } 1\end{array}$ & $\begin{array}{l}\text { Stereotactic bilateral } \\
\text { cingulotomy } 10-25 \mathrm{~mm} \\
\text { posterior to tip, } 6 \mathrm{~mm} \\
\text { above superior border } \\
\text { of anterior horn, } 12 \mathrm{~mm} \\
\text { from midline }\end{array}$ & NA \\
\hline
\end{tabular}


Table 2 (cont). Patient population, operative technique, and quantification of pain.

\begin{tabular}{|c|c|c|c|c|c|}
\hline & $\begin{array}{c}\text { Patient } \\
\text { demographics }\end{array}$ & $\begin{array}{l}\text { No. of } \\
\text { Patients } \\
\text { (neoplastic } \\
\text { + non- } \\
\text { neoplastic) }\end{array}$ & Cause of Pain & Operative technique & $\begin{array}{c}\text { Pain } \\
\text { Quantification }\end{array}$ \\
\hline $\begin{array}{l}\text { 6. Pillay and } \\
\text { Hassenbusch, } \\
1992\end{array}$ & $\begin{array}{l}\text { Age: range } 24- \\
69 \text { (mean } 51.9 \text { ) } \\
\text { Gender: not } \\
\text { stated }\end{array}$ & $10(8+2)$ & $\begin{array}{l}\text { - Neoplastic: All with bony } \\
\text { metastases: spindle cell carcinoma 1, } \\
\text { lung carcinoma 1, breast carcinoma } \\
3 \text {, rectal carcinoma 1, chordoma } 1 \text {, } \\
\text { myeloma 1 } \\
\text { - Non-neoplastic: } \\
\text { neurofibromatosis } 1 \text {, thalamic } \\
\text { stroke 1 }\end{array}$ & $\begin{array}{l}\text { Stereotactic bilateral } \\
\text { cingulotomy } 24 \mathrm{~mm} \\
\text { posterior to tip of } \\
\text { frontal horns under MRI } \\
\text { guidance, } 1 \text { lesion } 75 \mathrm{C} \text { for } \\
60 \mathrm{sec}\end{array}$ & NA \\
\hline $\begin{array}{l}\text { 7. Cohen et al., } \\
1999\end{array}$ & $\begin{array}{l}\text { Age: range } 40 \\
-58 \\
\text { Gender } 9 \mathrm{M} 3 \mathrm{~F}\end{array}$ & $12(0+12)$ & Noncerebral traumatic injury & $\begin{array}{l}\text { Stereotactic bilateral } \\
\text { anterior cingulotomy with } \\
\text { thermal probes creating } \\
\text { lesions } 5 \mathrm{~mm} \text { in diameter, } \\
\text { slightly lateral to midline }\end{array}$ & $\begin{array}{l}\text { Brief pain inventory } \\
\text { (10 point Likert } \\
\text { scale rated by } \\
\text { patient) }\end{array}$ \\
\hline $\begin{array}{l}\text { 8. Wilkinson et } \\
\text { al., } 1999\end{array}$ & $\begin{array}{l}\text { Age: range } 32 \\
-77 \\
\text { Gender: } 15 \mathrm{M} 8 \mathrm{~F}\end{array}$ & $23(0+23)$ & $\begin{array}{l}\text { Majority had lumbar or sciatic pain } \\
\text { related to "failed back syndrome" } \\
\text { or adhesive arachnoiditis. Also } \\
\text { phantom leg pain, venous occlusive } \\
\text { dz, ischemic bilateral leg pain, } \\
\text { "failed neck" pain, and atypical } \\
\text { facial pain }\end{array}$ & $\begin{array}{l}\text { Stereotactic } \\
\text { bilateral anterior } \\
\text { cingulotomy under air } \\
\text { ventriculography or CT } \\
\text { guidance. Radiofreq } \\
\text { thermocoagulation } 5 \mathrm{~mm} \\
\text { above roof of ventricle } \\
\text { and } 5 \mathrm{~mm} \text { lateral to } \\
\text { midline. with } 80 \mathrm{C} \text { for } 2 \\
\text { minutes. Then electrode } \\
\text { withdrawn } 1 \mathrm{~cm} \text { and } \\
\text { second lesion on each side }\end{array}$ & $\begin{array}{l}\text { Visual analog scale } \\
\text { with "excellent" ( }>6 \\
\text { points reduction), } \\
\text { "useful" ( }>2-5 \\
\text { points reduction) } \\
\text { and no relief }\end{array}$ \\
\hline $\begin{array}{l}\text { 9. Yen et al., } \\
2005\end{array}$ & $\begin{array}{l}\text { Age: Range } 35- \\
79, \text { mean } 58.3 \\
\text { years } \\
\text { Gender: 10M 5F }\end{array}$ & $22(15+7)$ & $\begin{array}{l}\text { Cancer: Mainly end stage } \\
\text { malignancy from bony mets. } 4 \\
\text { lung cancer, } 2 \text { breast cancer, } 2 \\
\text { HCC, } 2 \text { mesothelioma, } 1 \text { thyroid, } 1 \\
\text { esophageal, } 1 \text { ureteral, } 1 \text { lymphoma, } \\
1 \text { unknown primary } \\
\text { Non-Cancer: } 2 \text { diabetic neuropathy, } \\
2 \text { failed back surgery syndrome, } \\
2 \text { limb pain following sp injury, } 1 \\
\text { trigeminal neuralgia }\end{array}$ & $\begin{array}{l}\text { Stereotactic bilateral } \\
\text { anterior cingulotomy } \\
\text { under MRI guidance. } 24 \\
\text { mm posterior to frontal } \\
\text { horn Radiofrequency } \\
\text { thermocoagulation with } \\
\text { thermocoupled bipolar } \\
\text { lesion electrode }\end{array}$ & Visual analog scale \\
\hline $\begin{array}{l}\text { 10. Yen et al., } \\
2009\end{array}$ & $\begin{array}{l}\text { Age: } 40-72 \\
\text { years, mean } 64.4 \\
\text { years } \\
\text { Gender: } 6 \mathrm{M} 4 \mathrm{~F}\end{array}$ & $10(10+0)$ & $\begin{array}{l}\text { Terminally ill cancer (details not } \\
\text { provided) }\end{array}$ & $\begin{array}{l}\text { Identical to Yen et al., } \\
2005\end{array}$ & $\begin{array}{l}\text { McGill pain } \\
\text { questionnaire, with } \\
\text { good }>75 \% \text { pain } \\
\text { relief, fair } 25-75 \% \\
\text { pain relief, poor } \\
<25 \% \text { pain relief } \\
\end{array}$ \\
\hline $\begin{array}{l}\text { 11. Patel et al., } \\
2015\end{array}$ & $\begin{array}{l}\text { Age: } 38,45,51 \\
\text { Gender: } 0 \mathrm{M} 3 \mathrm{~F}\end{array}$ & $3(3+0)$ & $\begin{array}{l}\text { Liposarcoma of thigh with } \\
\text { metastasis to retroperitoneum and } \\
\text { deep muscles of back. Breast cancer } \\
\text { with metestasis to liver, lung, brain. } \\
\text { Colorectal Cancer with metastasis } \\
\text { to liver and lungs }\end{array}$ & $\begin{array}{l}\text { Bilateral anterior } \\
\text { cingulotomy via laser } \\
\text { interstitial thermal } \\
\text { therapy under MRI } \\
\text { guidance. } 2 \text { lesions } \\
\text { created bilaterally, } 2 \text { nd } \\
\text { lesion } 1 \mathrm{~cm} \text { above superior } \\
\text { to first, resulting in mean } \\
\text { lesion size } 1.44 \mathrm{~cm} 3\end{array}$ & $\begin{array}{l}\text { Brief pain inventory } \\
\text { (Pain Interference } \\
\text { scale and Pain } \\
\text { Severity Scale) }\end{array}$ \\
\hline
\end{tabular}


Table 3A. Patient outcomes.

\begin{tabular}{|c|c|c|c|c|c|}
\hline Article & $\begin{array}{l}\text { Patients } \\
\text { (neoplastic } \\
+ \text { non } \\
\text { neoplastic) }\end{array}$ & Neoplastic outcome & $\begin{array}{l}\text { Neoplastic } \\
\text { FU }\end{array}$ & Non-neoplastic outcome & $\begin{array}{l}\text { Non- } \\
\text { neoplastic FU }\end{array}$ \\
\hline $\begin{array}{l}\text { 1. Foltz and White, } \\
1962\end{array}$ & $16(6+10)$ & $\begin{array}{l}5 / 6 \text { patients with good- } \\
\text { excellent outcome }\end{array}$ & $\begin{array}{l}4 \text { days }-9 \\
\text { months }\end{array}$ & $\begin{array}{l}6 / 10 \text { patients with good- } \\
\text { excellent outcome }\end{array}$ & $\begin{array}{l}3 \text { months }-7 \\
\text { years }\end{array}$ \\
\hline $\begin{array}{l}\text { 2. Foltz and White, } \\
1968\end{array}$ & $35(11+24)$ & $\begin{array}{l}\text { 9/11 patients with good- } \\
\text { excellent outcome }\end{array}$ & Not stated & $\begin{array}{l}\text { 18/24 patients with good- } \\
\text { excellent outcome }\end{array}$ & 1 year -9 years \\
\hline 3. Faillace et al., 1971 & $9(7+2)$ & $3 / 7$ patients with pain relief & $\begin{array}{l}3 \text { days }-3 \\
\text { months }\end{array}$ & $1 / 2$ patients with pain relief & $>2$ years \\
\hline $\begin{array}{l}\text { 4. Hurt and Ballantine, } \\
1974\end{array}$ & $68(32+36)$ & $\begin{array}{l}\text { - } 18 / 32 \text { moderate to } \\
\text { complete relief at } 3 \text { months } \\
\text { or less } \\
-2 / 9 \text { moderate to complete } \\
\text { relief at }>3 \text { months }\end{array}$ & 4 days -6 years & $\begin{array}{l}\text { - } 16 / 36 \text { moderate to } \\
\text { complete relief at } 3 \text { months } \\
\text { or less } \\
\text { - } 16 / 36 \text { moderate to } \\
\text { complete relief at }>3 \text { months }\end{array}$ & $\begin{array}{l}6 \text { months }-9 \\
\text { years }\end{array}$ \\
\hline $\begin{array}{l}\text { 5. Voris and Whisler, } \\
1975\end{array}$ & $16(5+11)$ & $5 / 5$ relief to time of death & $1-12$ months & $\begin{array}{l}8 / 11 \text { relief at } 1-12 \text { months } \\
2 / 11 \text { relief at } 1 \text { year } \\
1 / 11 \text { at } 3 \text { years }\end{array}$ & $\begin{array}{l}1 \text { month to } 3 \\
\text { years }\end{array}$ \\
\hline $\begin{array}{l}\text { 6. Pillay and } \\
\text { Hassenbusch, } 1992\end{array}$ & $10(8+2)$ & $5 / 8$ good to excellent relief & 1 year & $1 / 2$ relief & 1 year \\
\hline 7. Cohen et al., 1999 & $12(0+12)$ & NA & NA & $8 / 12$ relief & 1 year \\
\hline 8. Wilkinson et al., 1999 & $23(0+23)$ & NA & NA & 18/23 useful-excellent relief & 1 year to 15 years \\
\hline 9. Yen et al., 2005 & $22(15+7)$ & $\begin{array}{l}-12 / 15 \text { meaningful- } \\
\text { significant relief at } 1 \text { week } \\
-9 / 15 \text { meaningful- } \\
\text { significant relief at } 1 \text { month } \\
-7 / 12 \text { meaningful- } \\
\text { significant relief (who } \\
\text { survived) at } 3 \text { months } \\
-5 / 10 \text { meaningful to } \\
\text { significant relief at } 6 \text { months }\end{array}$ & $\begin{array}{l}1 \text { week to } 6 \\
\text { months }\end{array}$ & $\begin{array}{l}-7 / 7 \text { meaningful-significant } \\
\text { relief at } 1 \text { week } \\
-5 / 7 \text { meaningful-significant } \\
\text { relief at } 1 \text { month } \\
-5 / 7 \text { meaningful-significant } \\
\text { at } 3 \text { months } \\
-5 / 7 \text { meaningful-significant } \\
\text { at } 6 \text { months } \\
-5 / 7 \text { meaningful-significant } \\
\text { at } 1 \text { year }\end{array}$ & 1 week to 1 year \\
\hline 10. Yen et al., 2009 & $10(10+0)$ & $\begin{array}{l}-6 / 10 \text { fair-good relief at } 1 \\
\text { week } \\
-5 / 10 \text { fair-good relief at } 1 \\
\text { month } \\
-6 / 10 \text { fair-good relief at } 3 \\
\text { months } \\
\end{array}$ & $\begin{array}{l}1 \text { week to } 3 \\
\text { months }\end{array}$ & NA & NA \\
\hline 11. Patel et al., 2015 & $3(3+0)$ & $\begin{array}{l}-3 \text { relief/3 at } 2 \text { weeks } \\
-1 \text { relief/2 at } 6 \text { week } \\
-1 \text { relief/2 at } 4 \text { months }\end{array}$ & $\begin{array}{l}2 \text { weeks to } 4 \\
\text { months }\end{array}$ & NA & NA \\
\hline TOTAL & $224(97+127)$ & 66/97 relief post-op & ------- & $83 / 127$ relief post-op & 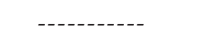 \\
\hline
\end{tabular}

$\mathrm{NA}=$ not available

this approach, of the 127 patients with non-neoplastic pain initially treated with anterior cingulotomy, at least $64(50 \%)$ had significant pain relief at 3 months and at least $47(37 \%)$ had significant pain relief at both 6 months and one year post-operatively.

\section{Reoperations}

A proportion of patients underwent reoperation (repeat cingulotomy) due to inadequate pain control after the initial surgical intervention. Importantly, the results of time-specific outcomes above include reoperations. Across all studies encompassing 224 patients, a total of 17 reoperations in 16 patients were identified across 5 reports $(9,15,17,19,23)$, corresponding to $7.6 \%$ of the total number of initial operations. Of the 17 reoperations, 10 reoperations $(9,15,17,19,23)$ were reported with sufficient detail from which individual patient successes of reoperation could be determined 
Table 3B. Patient outcomes with temporal resolution.

\begin{tabular}{|c|c|c|}
\hline Article & Neoplastic pain & Non-neoplastic pain \\
\hline $\begin{array}{l}\text { 1. Foltz and } \\
\text { White, } 1962\end{array}$ & $\begin{array}{l}\text { - } 4 \text { days: ( } 2 \text { excellent relief, } 3 \text { good relief, } 1 \text { fair } \\
\text { relief) / } 6 \text { patients } \\
-1 \text { month: }(2 \text { excellent relief, } 2 \text { good relief, } 1 \\
\text { fair relief) } / 5 \text { patients } \\
-5 \text { months: }(1 \text { excellent } 1 \text { fair }) / 2 \text { patients } \\
-9 \text { months: } 1 \text { excellent } / 1 \text { patient }\end{array}$ & $\begin{array}{l}-3 \text { months: ( } 3 \text { excellent relief, } 3 \text { good relief, } 2 \text { fair relief, } 2 \text { poor relief) / } 10 \\
\text { patients } \\
-5 \text { months: ( } 3 \text { excellent relief, } 3 \text { good relief, } 2 \text { fair relief, } 1 \text { poor relief) } / 9 \text { patients } \\
-6 \text { months: }(3 \text { excellent relief, } 2 \text { good relief, } 2 \text { fair relief, } 1 \text { poor relief) } / 8 \text { patients } \\
-1.5 \text { years: ( } 3 \text { excellent relief, } 2 \text { good relief, } 1 \text { fair relief, } 1 \text { poor relief) } / 7 \text { patients } \\
-2 \text { years: ( } 2 \text { excellent relief, } 2 \text { good relief, } 1 \text { fair relief, } 1 \text { poor relief at } 2 \text { years) } / 6 \\
\text { patients } \\
-3 \text { years: ( } 2 \text { excellent relief, } 2 \text { good relief, } 1 \text { fair relief) } / 5 \\
-4 \text { years: } 2 \text { excellent, } 2 \text { good) } / 4 \text { patients } \\
-4.5 \text { years: } 2 \text { excellent, } 1 \text { good } / 3 \text { patients } \\
-6 \text { years: } 1 \text { excellent } 1 \text { good } / 2 \text { patients } \\
-7 \text { years: } 1 \text { good } / 1 \text { patients }\end{array}$ \\
\hline $\begin{array}{l}\text { 2. Foltz and } \\
\text { White, } 1968\end{array}$ & $\begin{array}{l}\text { - FU unclear: ( } 5 \text { excellent, } 4 \text { good, } 2 \text { fair, } 0 \\
\text { poor, } 0 \text { worse) / } 11 \text { pts }\end{array}$ & - 1 year: ( 2 excellent, 5 good, 1 fair, 1 poor, 0 worse) / 9 pts \\
\hline $\begin{array}{l}\text { 3. Faillace et al., } \\
1971\end{array}$ & -3 days: ( 3 relief, 4 no relief)/ 7 patients & - >2 years: ( 1 relief, 1 no relief)/ 2 patients \\
\hline $\begin{array}{l}\text { 4. Hurt and } \\
\text { Ballantine, } 1974\end{array}$ & $\begin{array}{l}-<3 \text { months: ( } 3 \text { complete relief, } 9 \text { marked relief, } \\
6 \text { moderate relief, } 5 \text { slight relief, } 9 \text { no relief) / } 32 \\
\text { patients } \\
->3 \text { months: ( } 0 \text { complete, } 1 \text { marked, } 1 \\
\text { moderate, } 1 \text { slight, } 6 \text { none) } / 9 \text { patients }\end{array}$ & $\begin{array}{l}-<3 \text { months: }(2 \text { complete, } 6 \text { marked, } 8 \text { moderate, } 8 \text { slight, } 12 \text { none }) / 36 \\
\text { patients } \\
->3 \text { months: }(1 \text { complete, } 7 \text { marked, } 8 \text { moderate, } 8 \text { slight, } 12 \text { none }) / 36 \\
\text { patients }\end{array}$ \\
\hline $\begin{array}{l}\text { 5. Voris and } \\
\text { Whisler, } 1975\end{array}$ & - 1-12 months: 5 relief $/ 5$ at $1-12$ months & $\begin{array}{l}-1-12 \text { months: }(8 \text { relief. } 3 \text { no relief }) / 11 \text { pts } \\
-1 \text { year: }(2 \text { relief. } 9 \text { no relief) } / 11 \text { pts } \\
-3 \text { years: ( } 1 \text { relief. } 10 \text { no relief) / } 11 \text { pts }\end{array}$ \\
\hline $\begin{array}{l}\text { 6. Pillay and } \\
\text { Hassenbusch, } 1992\end{array}$ & $\begin{array}{l}-1 \text { year: } 4 \text { excellent, } 1 \text { good, } 1 \text { fair, } 2 \text { poor/ } \\
8 \text { pts }\end{array}$ & - 1 year: (1 good. 1 no relief) $/ 2$ pts \\
\hline $\begin{array}{l}\text { 7. Cohen et al., } \\
1999\end{array}$ & NA & - 1 year: (8 relief. 4 no relief)/12 pts \\
\hline $\begin{array}{l}\text { 8. Wilkinson et } \\
\text { al., } 1999\end{array}$ & NA & 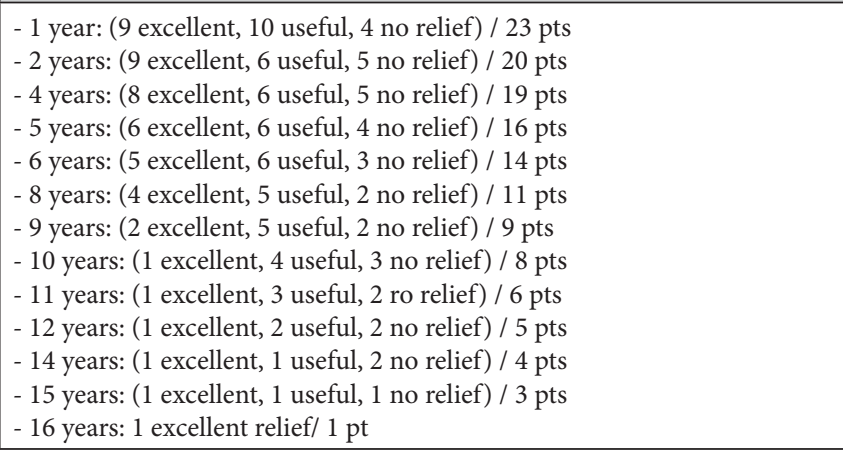 \\
\hline 9. Yen et al., 2005 & $\begin{array}{l}\text { - } 1 \text { week: ( } 8 \text { sig relief, } 4 \text { meaningful relief, } 3 \text { no } \\
\text { relief) /15 pts } \\
-1 \text { month: } 5 \text { sig relief, } 4 \text { meaningful relief, } 5 \\
\text { no relief) } / 15 \text { pts } \\
-3 \text { months: ( } 4 \text { sig relief, } 3 \text { meaningful relief, } 5 \\
\text { no relief) } / 12 \text { pts } \\
\text { - } 6 \text { months: ( } 2 \text { sig relief, } 3 \text { meaningful relief, } 5 \\
\text { no relief) } / 10 \text { pts }\end{array}$ & $\begin{array}{l}\text { - } 1 \text { week: } 6 \text { sig relief, } 1 \text { meaningful relief, } 0 \text { no relief) / } 7 \text { pts } \\
-1 \text { month: ( } 5 \text { sig relief, } 0 \text { meaningful relief, } 2 \text { no relief) / } 7 \text { pts } \\
-3 \text { months: ( } 4 \text { sig relief, } 1 \text { meaningful relief, } 2 \text { no relief) / } 7 \text { pts } \\
-6 \text { months: (4 sig relief, } 1 \text { meaningful relief, } 2 \text { no relief) / } 7 \text { pts } \\
-1 \text { year: (4 sig relief, } 1 \text { meaningful relief, } 2 \text { no relief) / } 7 \text { pts }\end{array}$ \\
\hline $\begin{array}{l}\text { 10. Yen et al., } \\
2009\end{array}$ & $\begin{array}{l}-1 \text { week ( } 4 \text { good relief, } 2 \text { fair relief, } 4 \text { no } \\
\text { improvement) } / 10 \\
-1 \text { month }(2 \text { good relief, } 3 \text { fair relief, } 5 \text { no } \\
\text { improvement) / } 10 \\
-3 \text { months ( } 4 \text { good relief, } 2 \text { fair relief, } 4 \text { no } \\
\text { improvement) / } 10\end{array}$ & NA \\
\hline $\begin{array}{l}\text { 11. Patel et al., } \\
2015\end{array}$ & $\begin{array}{l}-2 \text { weeks: } 3 \text { relief/ } 3 \text { pts } \\
-6 \text { weeks: } 1 \text { relief/ } 2 \text { pts } \\
-4 \text { months: } 1 \text { relief/ } 2 \text { pts }\end{array}$ & NA \\
\hline
\end{tabular}


Table 4. Percentage of patients with significant pain relief after operation.

\begin{tabular}{|l|c|c|c|}
\hline \multicolumn{1}{|c|}{ FU after Operation } & All patients & $\begin{array}{c}\text { Patients with pain due to } \\
\text { neoplastic source }\end{array}$ & $\begin{array}{c}\text { Patients with pain due to } \\
\text { non-neoplastic source }\end{array}$ \\
\hline Post-operatively & $149 / 224(67 \%)$ & $66 / 98(67 \%)$ & $83 / 127(65 \%)$ \\
\hline 3 months & $87 / 156(56 \%)$ & $23 / 44(52 \%)$ & $64 / 112(57 \%)$ \\
\hline 6 months & $59 / 94(63 \%)$ & $12 / 20(60 \%)$ & $47 / 74(64 \%)$ \\
\hline 1 year & $53 / 82(65 \%)$ & $6 / 9(67 \%)$ & $47 / 73(64 \%)$ \\
\hline
\end{tabular}

Table 5. Patient outcomes following reoperation.

\begin{tabular}{|c|c|c|c|}
\hline & Individual Patient data & $\begin{array}{c}\text { FU after } \\
\text { Reoperation }\end{array}$ & Reoperation technique \\
\hline Foltz and White, 1962 & $\begin{array}{l}1 \text { patient with poor pain relief after initial surgery } \\
\text { for } 3 \text { months, followed by good pain relief after } \\
\text { reoperation for } 4 \text { weeks }\end{array}$ & 4 weeks & Not reported \\
\hline Foltz and White, 1968 & $\begin{array}{l}3 \text { patients with poor pain relief after initial } \\
\text { surgery, followed by good or excellent pain relief } \\
\text { after reoperation }\end{array}$ & Not reported & $\begin{array}{l}\text { Placed more caudally in cingulum } \\
\text { bundle to enlarge lesions }\end{array}$ \\
\hline $\begin{array}{l}\text { Hurt and Ballantine, } \\
1974\end{array}$ & 7 reoperations in 6 patients. Details not reported & -1--- & -- \\
\hline Wilkinson et al., 1999 & $\begin{array}{l}\text { a) } 1 \text { patient with } 4 \text { months of no relief followed by } \\
\text { excellent pain relief } \\
\text { b) } 2 \text { patients with some but inadequate pain relief, } \\
\text { experiencing no change following reoperation } \\
\text { (post-reoperation followup }>1 \text { year) } \\
\text { c) } 2 \text { patients with excellent pain relief for whom } \\
\text { pain recurred. Following reoperation, one patient } \\
\text { had excellent pain relief for } 4 \text { years. Other patient } \\
\text { had inadequate followup post reoperation }\end{array}$ & $\begin{array}{l}\text { a) } 15 \text { years } \\
\text { b) }>1 \text { year } \\
\text { c) } 4 \text { years. Inadequate }\end{array}$ & $\begin{array}{l}\text { Same operative technique as initial } \\
\text { surgery. New lesions were placed to } \\
\text { enlarge prior lesions that appeared to be } \\
\text { small }(<15 \mathrm{~mm} \text { diameter) or adjacent to } \\
\text { prior lesions that did not seem optimally } \\
\text { placed }\end{array}$ \\
\hline Patel et al., 2015 & $\begin{array}{l}1 \text { patient who experienced recurrence after } 6 \\
\text { weeks of significant pain relief had significant } \\
\text { pain relief for } 4 \text { weeks (until dying from primary } \\
\text { disease). }\end{array}$ & 4 weeks & $\begin{array}{l}20 \mathrm{~mm} \text { anterior to initial lesion. } 3 \text { lesions } \\
\text { bilaterally (vs initial } 2 \text { lesions bilaterally), } \\
\text { resulting in mean lesion size } 2.73 \mathrm{~cm} 3 \\
\text { (vs initial } 1.44 \mathrm{~cm} 3 \text { mean lesion size) }\end{array}$ \\
\hline
\end{tabular}

$\mathrm{FU}=$ follow-up

(Table 5). Eight of these 10 reoperations (80\%) provided significant pain relief or improvement of symptoms post-operatively. Of these 10 reoperations, 6 reoperations had at least 4 weeks follow-up, of which $4(67 \%)$ were successful at the latest follow-up (2 of which included follow-up of more than 10 years). All 3 patients who had recurrence of pain after initial positive results had significant pain relief following reoperation.

\section{Effect of Lesion Location on Pain Relief}

Given the variance in the location of lesions within the anterior cingulate region across studies (Table 2), further analysis was done to evaluate if there was any effect of lesion location on pain relief. One variable that was reported across the majority of the articles ( $n=10$ ) was the distance in the $Y$ dimension posterior to the tip of the frontal horns (range 17.5 to 37.5 millimeters) (Table 2). Pooled linear regression analysis of post-operative outcome as a function of this distance was significant for $\mathrm{R}=-0.70(P<0.03)$, with regression coefficient of $2.1 \%$ mean decrement in positive outcomes (percent of patients significantly improved per study) per millimeter of distance posterior to the frontal horn tip.

\section{Adverse Events (Organic)}

No articles showed any mortality attributed to or associated with the surgery. Adverse events were characterized as the number of events per operation, where data were given (Table 6). Common transient adverse effects included urinary incontinence and confusion/disorientation, which subsided within days 
Table 6. Reported adverse events by article.

\begin{tabular}{|c|c|c|c|c|c|c|c|c|}
\hline \multirow{2}{*}{$\begin{array}{l}\text { Authors \& } \\
\text { Year }\end{array}$} & \multirow{2}{*}{$\begin{array}{l}\text { No. of } \\
\text { procedures }\end{array}$} & \multicolumn{4}{|l|}{ Transient Adverse Events } & \multicolumn{3}{|c|}{ Serious/Permanent Adverse Events } \\
\hline & & Event & $\begin{array}{l}\text { Time to } \\
\text { resolution }\end{array}$ & $\begin{array}{l}\text { No. of } \\
\text { events }\end{array}$ & $\%$ & Event & $\begin{array}{l}\text { No. of } \\
\text { events }\end{array}$ & $\%$ \\
\hline $\begin{array}{l}\text { Foltz and } \\
\text { White, } 1962\end{array}$ & 17 & $\begin{array}{l}\text { Mild elevation in temperature } \\
(100-101 F) \\
\text { Mild confusion } \\
\text { Change in affect (no flattening } \\
\text { or lethargy, however) }\end{array}$ & $\begin{array}{l}24 \text { hours } \\
1-2 \text { days } \\
\text { NA }\end{array}$ & $\begin{array}{l}\text { "often" } \\
\text { NA } \\
\text { NA }\end{array}$ & $\begin{array}{l}\text { NA } \\
\text { NA } \\
\text { NA }\end{array}$ & None & NA & NA \\
\hline $\begin{array}{l}\text { Foltz and } \\
\text { White, } 1968\end{array}$ & 37 & $\begin{array}{l}\text { Mild hemiparesis, transient } \\
\text { with full recovery } \\
\text { Urinary incontinence, } \\
\text { gradually controlled } \\
\text { Hypotension (70/50) for } 2 \\
\text { days } \\
\text { Disorientation to time for } \\
\text { 3-5 days }\end{array}$ & $\begin{array}{l}\text { NA } \\
\text { NA } \\
2 \text { days } \\
3-5 \text { days }\end{array}$ & $\begin{array}{l}1 \\
4 \\
1 \\
6\end{array}$ & $\begin{array}{l}2.7 \\
10.8 \\
2.7 \\
16.2\end{array}$ & $\begin{array}{l}\text { Hemiparesis worsened } \\
\text { to hemiplegia and } \\
\text { aphagia } \\
\text { Ventriculomegaly at } \\
\text { stage II operation } \\
\text { Tonic clonic seizure } \\
\text { lasting } 2 \text { days } \\
\text { Suicide* }\end{array}$ & $\begin{array}{l}1 \\
1 \\
1 \\
2\end{array}$ & $\begin{array}{l}2.7 \\
2.7 \\
2.7 \\
5.4\end{array}$ \\
\hline $\begin{array}{l}\text { Faillace et al., } \\
1971\end{array}$ & 9 & $\begin{array}{l}\text { Decrement in tapping test** } \\
\text { Decrement in porteus maze } \\
\text { testing }\end{array}$ & $\begin{array}{l}\text { NA } \\
2 \text { months }\end{array}$ & $\begin{array}{l}4 / 4^{\star *} \\
2\end{array}$ & $\begin{array}{l}100 \\
22.2\end{array}$ & None & NA & NA \\
\hline $\begin{array}{l}\text { Hurt and } \\
\text { Ballantine, } \\
1974\end{array}$ & 75 & $\begin{array}{l}\text { Headache and fever } \\
\text { commonly seen transiently in } \\
\text { first week after operation } \\
\text { transient bladder or bowel } \\
\text { incontinence } \\
\text { transient confusion }\end{array}$ & $\begin{array}{l}\sim 1 \text { week } \\
\text { NA } \\
\text { NA }\end{array}$ & $\begin{array}{l}\text { "common" } \\
\text { "less } \\
\text { common" } \\
\text { "less } \\
\text { common" }\end{array}$ & $\begin{array}{l}\text { NA } \\
\text { NA }\end{array}$ & $\begin{array}{l}\text { Guillain Barre } \\
\text { Syndrome }\end{array}$ & 1 & 1.3 \\
\hline $\begin{array}{l}\text { Voris and } \\
\text { Whisler, } 1975\end{array}$ & 16 & None & NA & NA & NA & $\begin{array}{l}\text { Hemiparesis } \\
\text { Prolonged stupor } \\
\text { Intracranial } \\
\text { hemorrhage }^{\star * *} \\
\end{array}$ & $\begin{array}{l}1 \\
2 \\
1\end{array}$ & $\begin{array}{l}6.3 \\
12.5 \\
6.3\end{array}$ \\
\hline $\begin{array}{l}\text { Pillay and } \\
\text { Hassenbusch, } \\
1992\end{array}$ & 10 & None & NA & NA & NA & None & NA & NA \\
\hline $\begin{array}{l}\text { Wilkinson et } \\
\text { al., } 1999\end{array}$ & 28 & $\begin{array}{l}\text { Flat affect and lack of } \\
\text { spontaneity } \\
\text { transient aphasia cleared in } \\
48 \text { hours. } \\
\text { Urinary incontinence } \\
\text { Repetitive hand washing } \\
\end{array}$ & $\begin{array}{l}\text { NA } \\
2 \text { days } \\
\text { NA } \\
\text { Several } \\
\text { days }\end{array}$ & $\begin{array}{l}1 \\
2 \\
3 \\
1\end{array}$ & $\begin{array}{l}3.6 \\
7.1 \\
10.7 \\
3.6\end{array}$ & $\begin{array}{l}\text { Intraoperative seizures } \\
\text { Postoperative seizures } \\
\text { Delayed seizures (4 of } \\
\text { which controlled with } \\
\text { anticonvulsants) }\end{array}$ & $\begin{array}{l}2 \\
2 \\
5\end{array}$ & \begin{tabular}{|l|}
7.1 \\
7.1 \\
17.9
\end{tabular} \\
\hline $\begin{array}{l}\text { Cohen et al., } \\
1999\end{array}$ & 8 (reported) & $\begin{array}{l}\text { Mutism } \\
\text { Akinesia/Bradykinesia/ } \\
\text { Psychomotor Slowing } \\
\text { Blunting of affect } \\
\text { Lethargy } \\
\end{array}$ & $\begin{array}{l}\text { Days } \\
\text { Days } \\
\text { Days } \\
\text { Days }\end{array}$ & $\begin{array}{l}3 \\
8 \\
6 \\
2\end{array}$ & $\begin{array}{l}37.5 \\
100 \\
75 \\
25\end{array}$ & $\begin{array}{l}\text { Changes in emotional } \\
\text { behavior, personality, } \\
\text { or cognitive ability } \\
\text { reported by families } 1 \\
\text { year postoperatively } \\
\end{array}$ & 6 & 75 \\
\hline $\begin{array}{l}\text { Yen et al., } \\
2005\end{array}$ & 22 & $\begin{array}{l}\text { Transient confusion } \\
\text { Upper GI bleed subsided w } \\
\text { med tx } \\
\text { Some attentional impairment } \\
\end{array}$ & $\begin{array}{l}\text { Few days } \\
\text { NA } \\
\text { NA }\end{array}$ & $\begin{array}{l}2 \\
2 \\
5\end{array}$ & $\begin{array}{l}9.1 \\
9.1 \\
22.7\end{array}$ & None & NA & NA \\
\hline $\begin{array}{l}\text { Yen et al., } \\
2009\end{array}$ & 10 & $\begin{array}{l}\text { Inappropriate uninhibited } \\
\text { speech } \\
\text { Impairment of focused } \\
\text { attention: time to complete } \\
\text { Stroop interference test } \\
\end{array}$ & $\begin{array}{l}2 \\
\mathrm{NA}\end{array}$ & $\begin{array}{l}2 \\
\mathrm{NA}\end{array}$ & $\begin{array}{l}20 \\
\mathrm{NA}\end{array}$ & None & NA & NA \\
\hline $\begin{array}{l}\text { Patel et al., } \\
2015\end{array}$ & 4 & None & NA & NA & NA & None & NA & NA \\
\hline
\end{tabular}

${ }^{\star}$ Patients were reported to have preoperative suicide tendency.

${ }^{* *}$ Indicates that future learning may be more difficult for repetitive motor tasks, such as knitting or shifting car gears. Only 4 patients were tested for this.

${ }^{* * *}$ Included in of pool results were patients who had combined surgeries with other ablative procedure. 
postoperatively. Serious or permanent adverse effects included seizure in $<5 \%$, hemiparesis in $<1 \%$, and personality change in $<1 \%$ of operations, reported across all studies, all of which primarily occurred in operations where magnetic resonance (MR)-guidance was not used.

\section{Adverse Effects (Neuropsychological)}

Three reports assessed neuropsychological deficits $(18,21,22)$. In their series of 9 patients, Faillace et al (18) performed subtle neuropsychological tests to assess frontal lobe dysfunction (e.g., Porteus Maze; tapping test) on 4 patients, and showed decrements in the tapping test (a non-verbal ordering test) postoperatively, suggesting difficulties with executive function and attention. The authors surmised that the deficit however is of uncertain clinical significance, but might make learning repetitive motor tasks such as knitting or manual shifting of a car more difficult. The authors did not report a time at which deficits may resolve. Difficulties with executive function and attention were further delved into by Cohen and colleagues (21), who also explored the time course of recovery after surgery. With respect to executive function, the authors assessed adaptive rate continuous performance testing and stroop performance, finding that executive function declines at 3 months from baseline but either recovered or nearly recovered at one year. On other tests, however, the authors found that spontaneous word production, object construction, and design fluency testing were impaired at 3 and 12 months, suggesting chronic impairments in intention and spontaneous response production. These results were in line with patients' families' reports of personality changes in patients, particularly continued behavioral passivity. The authors found no difference in language, visual, motor, or memory functions before and after anterior cingulotomy. Yen and colleagues in 2009 (22) performed similar testing as that of Cohen et al, finding deficits in stroop interference testing, confirming the attentional deficits after cingulotomy.

\section{Discussion}

\section{Summary of Evidence}

Anterior cingulotomy is effective in the treatment of chronic, medically refractory pain. First introduced by Le Beau in 1954 via open cingulectomy (36) and later adapted by Foltz and White in 1962 (9), anterior cingulotomy has been successfully performed in the treatment of chronic pain for decades. In recent years however, the use of anterior cingulotomy has declined in frequency due to a general move away from neuroablative procedures and towards nondestructive procedures such as neuromodulatory therapies and intrathecal opiate pumps (4-6). While these newer therapies are reversible, some patients remain refractory $(4,31)$ and they are associated with significant cost, perhaps not appropriate for certain populations of patients such as metastatic cancer patients whose life expectancy due to primary disease is already short.

The results of this comprehensive review suggest that ablative lesions of the anterior cingulate are effective in the treatment of chronic pain, both neoplastic and non-neoplastic sources. Adverse events related to anterior cingulotomy were few, commonly including transient post-operative confusion, urinary incontinence, headaches, or fever, all of which subsided in days post-operatively. Serious adverse events included hemiparesis, hemorrhage, and seizures, and by in large were seen in studies reporting cingulotomy with means other than magnetic resonance imaging (MRI) for intraoperative guidance. Neuropsychological adverse effects included deficits in executive function, attention, and spontaneous response production, all of which are commonly observed among patients with frontal lobe damage, thus suggesting that the fibers of the ACC are a part of the larger frontal-subcortical brain system (21).

In this review, a great proportion of cancer patients died prior to one-year follow-up due to the underlying primary disease, suggesting that palliative pain control in the long term may not be a critical consideration for such population of patients. Nevertheless, the current analysis suggests that long-term pain control is possible in both subsets of patients. A significant correlation was found between pain relief outcome and position of the lesion, with better outcomes found as the lesion target approached the tip of the frontal horns. This finding is consistent with a study in 8 patients at a single institution by Steele et al (37), who showed better outcomes in patients targeted more rostrally within the ACC when anterior cingulotomy was used to treat major depressive disorder. This correlation furthermore stands to reason given both human and animal evidence suggesting that the ACC exhibits functional segregation into a rostral emotional region, a posterior motor region, and an intermediate cognition region (38-40). Given the lack of systematic and uniform reporting of lesion location across all included studies however, further prospective studies using a single surgeon at a 
single institution are needed and can be corroborated further by tractography studies; however, the current results provide the first comprehensive analysis to date to provide any insight with respect to this issue as it relates to pain.

The reviewed evidence of reoperations supports that there may be benefit for repeat anterior cingulotomy in cases where initial pain relief is not adequate. The higher success rate of reoperation when compared to initial surgery suggests that cases where initial pain relief is inadequate may likely be due to inadequate initial lesioning or imprecise location of lesioning. The current evidence also suggests that reoperation is a viable option in patients who have resurgence of pain months after initial pain relief from the operation. However, given the small number of patients who had repeat cingulotomy after pain recurrence, a larger study involving repeat anterior cingulotomy for patients with pain recurrence would provide more insight with respect to this issue.

\section{Study Limitations}

There is significant heterogeneity in surgical technique evident amongst all reviewed studies. Method of ablation (electrocautery, radiofrequency ablation, laser ablation), number of lesions created, lesion location, and method of intraoperative guidance (ventriculography, computed tomography (CT)-guidance, MRIguidance) must be taken into account when generalizing to current neurosurgical practice. Hassenbusch et al (41), in 1990, first used MRI guidance for anterior cingulotomy, after time of which stereotactic anterior cingulotomy guided by MRI was readily performed. The current analysis (Table 2) suggests a trend towards outcomes improvement in operations using MRI guidance as opposed to other means of guidance. Thus, in the current era of MRI-guided stereotactic surgeries, outcomes after cingulotomy may be slightly greater than that suggested by the current review.

The method for quantifying and reporting pain relief was also variable across studies. Rather than clinical assessment or review of patient charts, some articles quantified pain using the visual analog scale, McGill pain questionnaire, or pain inventory scale. Some of these techniques, e.g., the visual analog scale, have been criticized as being crude and subjective (22). We believe however that the difficulty in generalizing across articles with difference in reporting technique is minimized by the standardization of what comprised in our review as "significant" pain relief.
This analysis included articles with different postoperative follow-up times. In the overall analysis of post-operative outcomes through time, care was taken to report at follow-up times that were reported by a plurality of included studies. If otherwise not included in the article, the next available time point was used to interpolate degree of pain relief at our reported time points (e.g., for articles not reporting outcomes at 3 months post surgery but reporting at 5 months, data from 5 month was interpolated to report outcomes at 3 months). Importantly, in no event was an earlier time point used to extrapolate to a later time point - leaving all outcome calculations conservative. Percentages of patients with relief appeared to increase from 3 months to one year. However, this is in part due to patients, particularly cancer patients, with none-poor relief often dying before counterparts with greater degrees of pain relief (1). Moreover, studies with poorer outcomes relative to other studies often had inadequate follow-up times (18) or inadequate temporal resolution of postoperative follow-up (19) necessary to be included in the analysis at later time points. This may falsely elevate pain relief at later post-operative times. Thus, patients who were lost to follow-up were additionally assumed to be failures, providing a further conservative measure of proportions of patients with significant pain relief.

Finally, all studies were solely observational studies without controls. Hunt and Ballatine in 1974 (19) suggested that the number of patients who experienced significant pain relief following this surgery is great enough to suggest that the benefit after the operation is more than that which would be expected by placebo effect alone. Furthermore, as Bourne et al previously pointed out in 2013 (42), the ethical considerations surrounding randomization of patients with such degree of illness to sham surgery likely renders this analysis as the best available class of data on this topic. However, we acknowledge that placebo can be a significant factor in assessing outcomes of pain interventions (43-46) and should be considered in future studies.

\section{Conclusions}

Despite decreased utilization in recent years, anterior cingulotomy is an effective neurosurgical intervention in the treatment of pain, particularly under MRI guidance, and carries little risk of permanent or serious adverse effects. Despite the reported success rates in the literature, most of these studies were performed in an era prior to neuromodulatory therapies (e.g., spinal cord stimulation and intrathecal pain management). 
It is therefore unclear how effective anterior cingulotomy may be in patients who have failed these other therapies. Given the overall success rate in patients who had otherwise failed other pain management op- tions, studies are now needed to re-evaluate the role of anterior cingulotomy in patients with medically and neuromodulatory-refractory pain.

\section{References}

1. Yen CP, Kung SS, Su YF, Lin WC, Howng $\mathrm{SL}$, Kwan AL. Stereotactic bilateral anterior cingulotomy for intractable pain. J Clin Neurosci 2005; 12:886-890.

2. Pillay PK, Hassenbusch SJ. Bilateral MRI-guided stereotactic cingulotomy for intractable pain. Stereotact Func Neurosurg 1992; 59:33-38.

3. Harsh V, Viswanathan A. Surgical/radiological interventions for cancer pain. Curr Pain Headache Rep 2013; 17:331.

4. Cetas JS, Saedi T, Burchiel KJ. Destructive procedures for the treatment of nonmalignant pain: A structured literature review. J Neuorosurg 2008; 109:389-404.

5. Raslan AM, Cetas JS, McCartney S, Burchiel KJ. Destructive procedures for control of cancer pain: The case for cordotomy. J Neurosurg 2011; 114:155-170.

6. Loyd RD, Ball PA, Fanciullo GJ. Surgical procedures for intractable cancer pain. Tech Reg Anesth Pain Manag 2005; 9:167-176.

7. Boccard SG, Fernandes H, Jbabdi S, Hartvelt TJ, Kringelbach ML, Quaghebeur G, Moir L, Mancebo VP, Pereira EA, Fitzgerald J, Green AL, Stein J, Aziz TZ. A tractography study of deep brain stimulation of the anterior cingulate cortex in chronic pain: A key to improve the targeting. World Neurosurg 2016; 86:361-370.

8. Melzack R. From the gate to the neuromatrix. Pain 1999; Suppl 6:S121-S126.

9. Foltz EL, White Jr LE. Pain "relief" by frontal cingulumotomy. J Neurosurg 1962; 19:89-100.

10. Rolls ET, O’Doherty J, Kringelbach ML, Francis S, Bowtell R, McGlone F. Representations of pleasant and painful touch in the human orbitofrontal and cingulate cortices. Cereb Cortex 2003; 13:308-317.

11. Fodstad H, Strandman E, Karlsson B, West KA. Treatment of chronic obsessive compulsive states with stereotactic anterior capsulotomy or cingulotomy. Acta Neurochir (Wien) 1982; 62:1-23.

12. Ballantine HT, Bouckoms AJ, Thomas
EK, Giriunas IE. Treatment of psychiatric illness by stereotaxic cingulotomy. Biol Psychiatry 1987; 22:807-819.

13. Ballantine HT, Jr. Historical overview of psychosurgery and its problematic. Acta Neurochir Suppl 1988; 44:125-128.

14. Cosgrove G, Ballantine HJ. Cingulotomy in psychotherapy. In: Gildenberg P, Tasker R (eds). Textbook of Stereotactic and Functional Neurosurgery. McGrawHill, New York, 1997, pp 1965-1970.

15. Wilkinson HA, Davidson KM, Davidson RI. Bilateral anterior cingulotomy for chronic noncancer pain. Neurosurgery 1999; 45:1129-1136.

16. Brown LT, Mikell CB, Youngerman BE, Zhang Y, McKhann GM, Sheth SA. Dorsal anterior cingulotomy and anterior capsulotomy for severe, refractory obsessive-compulsive disorder: A systematic review of observational studies. J Neurosurg 2015:1-13.

17. Foltz EL, White LE. The role of rostral cingulumotomy in "pain" relief. Int J Neurol 1968; 6:353-373.

18. Faillace LA, Allen RP, McQueen JD, Northrup B. Cognitive deficits from bilateral cingulotomy for intractable pain in man. Dis Nerv Syst 1971; 32:171-175.

19. Hurt RW, Ballantine Jr HT. Stereotactic anterior cingulate lesions for persistent pain: A report on 68 cases. Clin Neurosurg 1974; 21:334-351.

20. Voris HC, Whisler WW. Results of stereotaxic surgery for intractable pain. Confin Neurol 1975; 37:86-96.

21. Cohen RA, Kaplan RF, Moser DJ, Jenkins MA, Wilkinson $\mathrm{H}$. Impairments of attention after cingulotomy. Neurology 1999; 53:819-824.

22. Yen CP, Kuan CY, Sheehan J, Kung SS, Wang CC, Liu CK, Kwan AL. Impact of bilateral anterior cingulotomy on neurocognitive function in patients with intractable pain. J Clin Neurosci 2009; 16:214-219.

23. Patel NV, Agarwal N, Mammis A, Danish SF. Frameless stereotactic magnetic resonance imaging-guided laser interstitial thermal therapy to perform bilateral an- terior cingulotomy for intractable pain: Feasibility, technical aspects, and initial experience in 3 patients. Neurosurgery 2015; 11:17-25.

24. Vogt BA, Finch DM, Olson CR. Functional heterogeneity in cingulate cortex: The anterior executive and posterior evaluative regions. Cereb Cortex 1992; 2:435-443.

25. Dowman R, Schell S. Evidence that the anterior cingulate and supplementary somatosensory cortices generate the pain-related negative difference potential. Neurophysiol Clin 1999; 110:2117-2126.

26. Talbot JD, Marrett S, Evans AC, Meyer E, Bushnell MC, Duncan GH. Multiple representations of pain in human cerebral cortex. Science 1991; 251:1355-1358.

27. Casey KL, Minoshima S, Berger KL, Koeppe RA, Morrow TJ, Frey KA. Positron emission tomographic analysis of cerebral structures activated specifically by repetitive noxious heat stimuli. ] Neurophysiol 1994; 71:802-807.

28. Vogt BA, Derbyshire S, Jones AK. Pain processing in four regions of human cingulate cortex localized with co-registered PET and MR imaging. Eur ] Neurosci 1996; 8:1461-1473.

29. Lenz FA, Rios M, Zirh A, Chau D, Krauss G, Lesser RP. Painful stimuli evoke potentials recorded over the human anterior cingulate gyrus. J Neurophysiol 1998; 79:2231-2234.

30. Zhuo M. Long-term potentiation in the anterior cingulate cortex and chronic pain. Philos Trans $R$ Soc Lond B Biol Sci 2014; 369.

31. Coffey RJ, Lozano AM. Neurostimulation for chronic noncancer pain: An evaluation of the clinical evidence and recommendations for future trial designs. J Neurosurg 2006; 105:175-189.

32. Manchikanti L. Evidence-based medicine, systematic reviews, and guidelines in interventional pain management part I: Introduction and general considerations. Pain Physician 2008; 11:161-186.

33. Moher D, Liberati A, Tetzlaff J, Altman 
DG. Preferred reporting items for systematic reviews and meta-analyses: The PRISMA statement. Ann Intern Med 2009; 151:264-269, w264.

34. Barthas F, Sellmeijer J, Hugel S, Waltisperger E, Barrot M, Yalcin I. The anterior cingulate cortex is a critical hub for pain-induced depression. Biol Psychiatry 2015; 77:236-245.

35. Wilson $\mathrm{DH}, \mathrm{Chang} \mathrm{AE}$. Bilateral anterior cingulectomy for the relief of intractable pain. Report of 23 patients. Confin Neurol 1974; 36:61-68.

36. Le Beau J. Anterior cingulectomy in man. J Neurosurg 1954; 11:268-276.

37. Steele JD, Christmas D, Eljamel MS, Matthews K. Anterior cingulotomy for major depression: Clinical outcome and relationship to lesion characteristics. Biol Psychiatry 2008; 63:670-677.

38. Bush G, Luu P, Posner MI. Cognitive and emotional influences in anterior cingulate cortex. Trends Cogn Sci 2000; 4:215-222.

39. Steele JD, Lawrie SM. Segregation of cognitive and emotional function in the prefrontal cortex: A stereotactic meta- analysis. Neuroimage 2004; 21:868-875.

40. Mega $M$, Cummings J. The cingulate and cingulate syndromes. In: Trimble $\mathrm{M}$, Cummings J (eds). Contemporary Behavioural Neurology. ButterworthHeinemann, Oxford, England, 1997, pp 189-214.

41. Hassenbusch SJ, Pillay PK, Barnett GH. Radiofrequency cingulotomy for intractable cancer pain using stereotaxis guided by magnetic resonance imaging. Neurosurgery 1990; 27:220-223.

42. Bourne SK, Sheth SA, Neal J, Strong C, Mian MK, Cosgrove GR, Eskandar EN Dougherty DD. Beneficial effect of subsequent lesion procedures after nonresponse to initial cingulotomy for severe, treatment-refractory obsessive-compulsive disorder. Neurosurgery 2013; 72:196202; discussion 202.

43. Amanzio M, Benedetti F, Porro CA, Palermo S, Cauda F. Activation likelihood estimation meta-analysis of brain correlates of placebo analgesia in human experimental pain. Hum Brain Mapp 2013; 34:738-752.

44. Jarcho JM, Feier NA, Labus JS, Naliboff
B, Smith SR, Hong JY, Colloca L, Tillisch K, Mandelkern MA, Mayer EA, London ED. Placebo analgesia: Self-report measures and preliminary evidence of cortical dopamine release associated with placebo response. Neuroimage Clin 2016; 10:107-114.

45. Dworkin RH, Turk DC, Wyrwich KW, Beaton D, Cleeland CS, Farrar JT, Haythornthwaite JA, Jensen MP, Kerns RD, Ader DN, Brandenburg N, Burke LB, Cella D, Chandler J, Cowan P, Dimitrova R, Dionne R, Hertz S, Jadad AR, Katz NP, Kehlet H, Kramer LD, Manning DC, McCormick C, McDermott MP, McQuay HJ, Patel S, Porter L, Quessy S, Rappaport BA, Rauschkolb C, Revicki DA, Rothman M, Schmader KE, Stacey BR, Stauffer JW, von Stein T, White RE, Witter J, Zavisic $S$. Interpreting the clinical importance of treatment outcomes in chronic pain clinical trials: IMMPACT recommendations. J Pain 2008; 9:105-121.

46. Manchikanti L, Giordano J, Fellows B, Hirsch JA. Placebo and nocebo in interventional pain management: A friend or a foe or simply foes? Pain Physician 2011; 14:E157-E175. 